\title{
CAPÍTULO 03: MAPEAMENTO DA PRODUTIVIDADE DO GIRASSOL (Helianthus annuus L.) E DOS ATRIBUTOS DO SOLO EM ÁREA DE PLANTIO DIRETO
}

\author{
CAPÍTULO 03: MAPEO DE LA PRODUCTIVIDAD DEL GIRASOL (Helianthus \\ annuus L.) Y LOS ATRIBUTOS DEL SUELO EN ZONA DE SEMBRANZA
}

\author{
CHAPTER 03: MAPPING OF SUNFLOWER (Helianthus annuus L.) \\ PRODUCTIVITY AND SOIL ATTRIBUTES IN NO-TILLAGE AREA
}

\author{
Leticia de Almeida Sorano1; Matheus Katriel dos Santos Araujo²; Eliude Alice Neves³; Grazieli Suszek ${ }^{4}$; Mauro \\ de Lima $^{5}$
}

DOI: https://doi.org/10.31692/978-65-88970-07-2.36-54

\begin{abstract}
RESUMO
O Girassol (Helianthus annuus L.) apresenta destaque mundialmente, como quinta oleaginosa em produção de grãos e entre as quatro principais culturas produtoras de óleo comestível. Além disso, possui características agronômicas importantes, como maior resistência à seca, ao frio e ao calor do que a maioria das espécies normalmente cultivadas no Brasil. Desta forma este trabalho tem por objetivo realizar o mapeamento da variabilidade espacial da produtividade do girassol e de atributos físico e químicos do solo em área de plantio direto no município de Nova Andradina - MS. Esse trabalho foi desenvolvido em área experimental pertencente ao Instituto Federal de Mato Grosso do Sul - campus de Nova Andradina/MS, onde foi realizada a semeadura do Girassol utilizando-se a cultivar BRS 324. Para a realização do trabalho, foi elaborado um Grid regular com malha amostral de 9x20 m, totalizando 12 pontos amostrais, georreferenciados utilizando-se GPS 76CSx da Garmin. Em cada ponto amostral foi realizada uma amostragem de solo na profundidade de $0-0,20 \mathrm{~m}$, análise de resistência do solo à penetração (RP), umidade do solo, colheita e peso de mil aquênios (PMA). Os dados obtidos das análises foram submetidos à análise estatística descritiva, para elaboração dos mapas foi utilizado o Software ArcGIS usando interpolação pelo Inverso do quadrado da distância (IQD). Foi possível identificar variabilidade espacial na área experimental, através do mapeamento da produtividade e dos atributos físicos e químicos do solo. Além disso, observou-se correlação significativa entre a produtividade o peso de mil aquênios e os atributos de $\mathrm{pH}, \mathrm{H}+\mathrm{Al}, \mathrm{B}, \mathrm{Fe}$ e Mn.

Palavras-Chave: Agricultura de precisão, Fertilidade do solo, Fisica do solo.
\end{abstract}

\section{RESUMEN}

El Girasol (Helianthus annuus L.) se destaca en todo el mundo, como finca oleaginosa en la producción de cereales y entre los cuatro principales cultivos productores de aceite comestible. Además, tiene importantes características agronómicas, como mayor resistencia a la sequía, al frío y al calor que la mayoría de las especies que se cultivan normalmente en Brasil. De esta manera, este trabajo tiene como objetivo mapear la variabilidad espacial de la productividad del girasol y los atributos físicos y químicos del suelo en un área de labranza cero en el municipio de Nova Andradina MS. Este trabajo se desarrolló en un área experimental perteneciente al Instituto Federal de Mato

\footnotetext{
${ }^{1}$ Bacharelado em Agronomia, Instituto Federal de Educação, Ciência e Tecnologia de Mato Grosso do Sul, leticia.sorano@gmail.com

${ }^{2}$ Bacharelado em Agronomia, Instituto Federal de Educação, Ciência e Tecnologia de Mato Grosso do Sul, matheus.araujo@novaandradina.org

${ }^{3}$ Tecnologia em Produção de Grãos, Instituto Federal de Educação, Ciência e Tecnologia de Mato Grosso do Sul, eliude.neves@novaandradina.org

${ }^{4}$ Professora Doutora em Engenharia Agrícola, Instituto Federal de Educação, Ciência e Tecnologia de Mato Grosso do Sul, grazieli.suszek@ifms.edu.br [36]

${ }^{5}$ Professor Especialista em Engenharia Agrícola, Instituto Federal de Educação, Ciência e Tecnologia de Mato Grosso do Sul, mauro.lima@ifms.edu.br
} 
Grosso do Sul - campus de Nova Andradina / MS, donde se realizó la siembra de Girasol con el cultivar BRS 324. Para el trabajo se elaboró una cuadrícula regular con una cuadrícula de muestreo de 9x20 m, totalizando 12 puntos de muestreo, georreferenciados mediante GPS 76CSx de Garmin. En cada punto de muestreo se realizó muestreo de suelo a una profundidad de $0-0,20 \mathrm{~m}$, análisis de resistencia del suelo a la penetración (RP), humedad del suelo, cosecha y peso de mil aquenios (PMA). Los datos obtenidos de los análisis fueron sometidos a análisis estadístico descriptivo, para la elaboración de los mapas se utilizó el Software ArcGIS mediante la interpolación inversa del cuadrado de distancia (IQD). Sin embargo, fue posible identificar la variabilidad espacial en el área experimental, al mapear la productividad y los atributos físicos y químicos del suelo. Se observó una correlación significativa entre la productividad, el peso de mil aquenios y los atributos de $\mathrm{pH}, \mathrm{H}+\mathrm{Al}, \mathrm{B}, \mathrm{Fe}$ y Mn.

Palabras Clave: Agricultura de precisión, Fertilidad del suelo, Física del suelo.

\section{ABSTRACT}

The Sunflower (Helianthus annuus L.) is highlighted worldwide, as an oilseed farm in grain production and among the four main crops producing edible oil. In addition, it has important agronomic characteristics, such as greater resistance to drought, cold and heat than most species normally grown in Brazil. In this way, this work aims to map the spatial variability of sunflower productivity and physical and chemical attributes of the soil in a no-tillage area in the municipality of Nova Andradina - MS. This work was developed in an experimental area belonging to the Federal Institute of Mato Grosso do Sul - campus of Nova Andradina / MS, where the Sunflower sowing was carried out using the BRS 324 cultivar. For the work, a regular grid with a 9x20 m sampling grid was elaborated, totaling 12 sampling points, georeferenced using GPS 76CSx from Garmin. At each sampling point, soil sampling was performed at a depth of $0-0.20 \mathrm{~m}$, analysis of soil resistance to penetration (RP), soil moisture, harvest and weight of a thousand achenes (PMA). The data obtained from the analyzes were elaborated to the descriptive statistical analysis, for the elaboration of the maps it was used in the ArcGIS Software using Interpolation by Inverse of the square of the distance (IQD). It was possible to identify the spatial variability in the experimental area, by mapping the productivity and the physical and chemical attributes of the soil. In addition, we observed a correlation between the productivity of the weight of a thousand achenes and the attributes of $\mathrm{pH}, \mathrm{H}+\mathrm{Al}, \mathrm{B}, \mathrm{Fe}$ and $\mathrm{Mn}$.

Keywords: precision agriculture, Soil fertility, Soil physics.

\section{INTRODUÇÃO}

O Girassol (Helianthus annuus L.) é uma dicotiledônea de ciclo anual, pertencente a família Compositae, originária do continente norte americano. Atualmente, o girassol é cultivado em todos os continentes, em área que atinge aproximadamente 24 milhões de hectares. Além disso, apresenta destaque mundialmente, como quinta oleaginosa em produção de grãos e entre as quatro principais culturas produtoras de óleo comestível (RIBAS, 2009).

Essa oleaginosa ainda apresenta características agronômicas importantes, como maior resistência à seca, ao frio e ao calor do que a maioria das espécies normalmente cultivadas no Brasil. Também obtém ampla adaptabilidade às diferentes condições edafoclimáticas e seu rendimento é pouco influenciado pela latitude, pela altitude e pelo fotoperíodo. Devido a essas características, apresenta-se como umą̧pção nos sistemas de rotação e sucessão de culturas nas regiões produtoras de grãos (CASTRO et al., 1996). 
No entanto, o Brasil é ainda um pequeno produtor de girassol com uma área cultivada de 111,3 mil hectares, produção de 147,1 mil toneladas e produtividade média de $1323 \mathrm{~kg} / \mathrm{ha}$ (LIRA et al., 2010). Segundo Gazzola et al. (2012), em lavouras mais tecnificadas a produtividade do girassol pode alcançar até $2400 \mathrm{~kg} / \mathrm{ha}$.

Além disso, às áreas utilizadas nas atividades agrícolas não são obrigatoriamente uniformes, onde dentro de uma mesma gleba existirão diferenças no solo e relevo que podem significar a demanda por tratamentos diferenciados (MOLIN, AMARAL e COLAÇO, 2015).

Do ponto de vista de Bernardi et al. (2014), é indispensável utilizar técnicas e métodos que indiquem ao produtor onde ocorre a variabilidade em sua propriedade agrícola, para que desta maneira possa se ter uma melhor produtividade.

De acordo com Comunello et. al. (2014), a agricultura de precisão trata-se de um conjunto de ferramentas e de conceitos para gerenciar a variabilidade espacial de atributos do solo, da planta e do ambiente, visando a obtenção de elevadas produtividades e maior eficiência de uso de práticas e insumos, e a redução do impacto ambiental.

Já segundo Gimenez e Molin (2004), o mapeamento da produtividade é considerado por muitos pesquisadores como sendo uma parte essencial da agricultura de precisão. Thylén et al. (1997) consideraram que o mapa de produtividade é um dos métodos mais corretos para estimar a heterogeneidade de uma lavoura. Um mapa de produtividade evidencia regiões com alta e baixa produtividade, mas não explica a causa de tal variação, que pode ser devido a doenças, a deficiências, a desequilíbrios químicos ou estresse hídrico e se a causa específica não é determinada, nenhuma resposta ao gerenciamento pode ser obtida (Lark \& Stafford, 1997).

Desta forma este trabalho tem por objetivo realizar o mapeamento da variabilidade espacial da produtividade do girassol e de atributos físico e químicos do solo em área de plantio direto no município de Nova Andradina - MS.

\section{FUNDAMENTAÇÃO TEÓRICA}

O girassol é uma cultura que tem sua área em constante oscilação no Brasil, sendo que na safra 2018/2019, a área plantada foi em torno de 95 mil hectares e a produtividade de 1561 $\mathrm{kg} /$ ha (Conab, 2019). O fator mais limitante para a cultura na maioria das regiões produtoras é a ocorrência de doenças, que é estimada mundialmente como sendo responsável por perdas anuais da ordem de $12 \%$ da produção (LEITE, 2016). 
A contemplação de diferentes setores econômicos, tais como a alimentação humana e animal, produção de biocombustível, e sua fácil adaptabilidade às diferentes regiões de cultivo, têm promovido a ascensão produtiva da cultura no âmbito nacional e internacional (OLIVEIRA et. al., 2017).

O girassol é uma espécie sensível à acidez do solo, geralmente os solos do Cerrado, em sua maioria, apresentam solos de baixa capacidade de troca de cátions (CTC), com acidez elevada e com baixo teor de nutrientes, especialmente de P (MONTEZANO, CORAZZA e MURAOKA, 2006).

Além disso, existe certa heterogeneidade dos atributos químicos e físicos de um solo, mesmo em uma área considerada uniforme, segundo suas características visíveis de campo, tais como: topografia, cor do solo e vegetação (MONTEZANO, CORAZZA e MURAOKA, 2006). A variabilidade espacial nos atributos do solo pode ser influenciada pelos seus fatores intrínsecos (fatores de formação, que são o material de origem, relevo, clima, organismos e tempo) e pelos fatores extrínsecos, normalmente relacionados com as práticas de manejo (CARVALHO, TAKEDA e FREDDI, 2003).

De acordo com BRASIL (2009a), Agricultura de precisão (AP) é o conjunto de técnicas e metodologias que visam otimizar o manejo de cultivos e a utilização dos insumos agrícolas, proporcionando máxima eficiência econômica. As ferramentas de AP permitem o uso racional dos fertilizantes e agrotóxicos garantindo a redução dos impactos ambientais decorrentes da atividade agrícola.

\section{METODOLOGIA}

O experimento foi conduzido em uma área experimental com as dimensões $18 \mathrm{~m}$ de largura, $120 \mathrm{~m}$ de comprimento, totalizando $2160 \mathrm{~m}^{2}$, localizado no município de Nova Andradina / MS, pertencente ao Instituto Federal de Educação, Ciência e Tecnologia de Mato Grosso do Sul, Situada nas coordenadas de $22^{\circ} 04^{\prime} 56,33^{\prime \prime}$ S e $53^{\circ} 28^{\prime} 08,38^{\prime \prime}$ O, conforme Figura 01. O clima da região é classificado, segundo Köeppen, como tropical subtropical úmido e mesotérmico, possuindo altitude média de $380 \mathrm{~m}$, com índices pluviométricos superiores a $1100 \mathrm{~mm}$ anuais e o solo da área experimental é classificado como Latossolo Vermelho de textura arenosa conforme Santos et al. (2018), o solo da área é composto por $82,68 \%$ areia, $2,6 \%$ silte e $14,72 \%$ de argila.

Figura 01. Localização do experimento em Nova Andradina/MS 


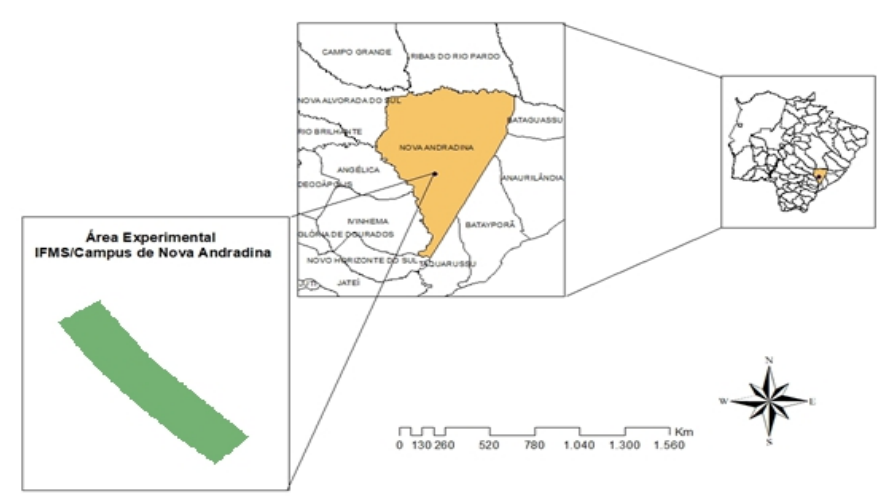

Fonte: Própria (2020).

Para a realização do trabalho, foi elaborado um Grid regular com dimensão de malha amostral de 9x20 m, onde foram escolhidos 12 pontos amostrais (Figura 02), georreferenciados utilizando-se GPS 76CSx da Garmin. Em cada ponto amostral foi realizada coleta de amostras de solo, análise de resistência do solo à penetração e colheita.

Figura 02. Distribuição dos pontos amostrais na área na experimental.

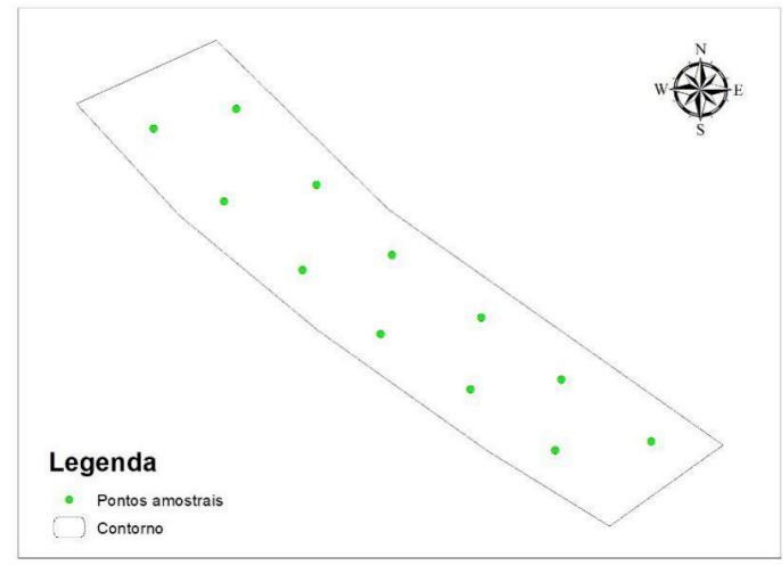

Fonte: Própria (2020).

A amostragem de solo foi realizada em abril de 2019, para amostragem de solo, coletou-se em cada ponto uma amostra composta de 8 sub-amostras (raio de $5 \mathrm{~m}$ ) na profundidade de $0-20 \mathrm{~cm}$, utilizando-se um trado holandês (Figura 03), sendo as mesmas, foram identificadas e encaminhadas para laboratório para análise química, conforme Belletti (2009) e Cherubin et al. (2016).

Figura 03. Coleta das amostras de solo, utilizando trado holandês. 


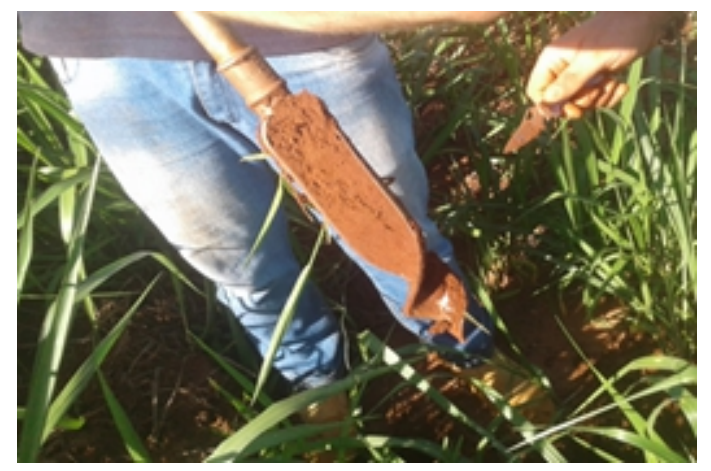

Fonte: Própria (2020).

A avaliação de resistência do solo à penetração foi realizada em Outubro de 2019, utilizando-se o penetrômetro eletrônico, modelo FALKER PenetroLOG - PLG1020, com aptidão eletrônica para aquisição de dados. Este penetrômetro foi configurado para registrar leituras a cada $0,01 \mathrm{~m}$ de incremento de profundidade, trabalhando em velocidade de penetração constante. Os dados referentes ao penetrômetro Falker foram extraídos da memória digital e analisados a uma profundidade máxima de $0,2 \mathrm{~m}$. Para o processamento dos dados de resistência à penetração, foi utilizado o Software PenetroLOG.

Durante as avaliações de resistência do solo à penetração (RP), também foram coletadas amostras de solo na profundidade de 0-20 cm, para determinação da umidade através do método gravimétrico (Santos et al., 2018), assim as amostras foram coletadas utilizando trado holandês (Figura 03), posteriormente as amostras foram envolvidas em papel alumínio, e levadas ao laboratório de solos e nutrição de plantas do IFMS, campus Nova Andradina-MS, para pesagem e secagem em estufa a $105^{\circ} \mathrm{C}$ por 24 horas (Figura 04).

Figura 04. Amostras de solo em estufa para realização da secagem.

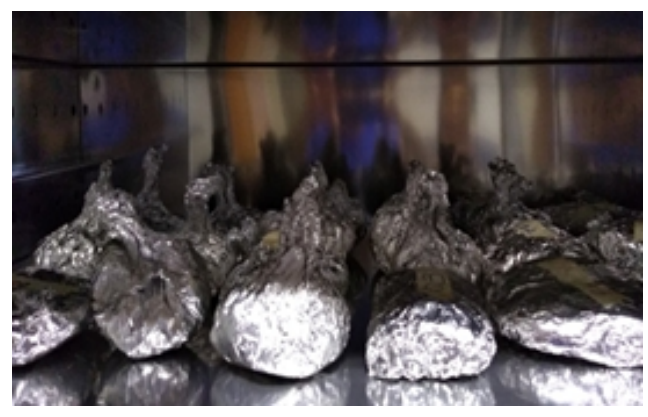

Fonte: Própria (2020).

A semeadura do girassol foi realizada no dia 21 de novembro de 2019, em condições ideais de umidade do solo, não apresentando problemas na germinação e emergência da cultura. A cultivar de girassol escolhida parp4P] experimento foi a BRS 324, por apresentar adaptabilidade à região, alto teor de óleo nos aquênios e tolerância à seca, ao frio e ao calor. 
A recomendação de adubação foi realizada com base no resultados da análise química do solo, conforme recomendação proposta por Sousa e Lobato (2004). As aplicações de herbicidas, inseticidas e fungicidas foram realizadas de acordo com a necessidade de tais controles, levando sempre em consideração o custo benefício das aplicações e condições climáticas.

A colheita foi realizada pelo método manual, no qual foi confeccionado uma armação, com dimensões de $0,50 \mathrm{~m}$ x $0,50 \mathrm{~m}\left(0,25 \mathrm{~m}^{2}\right)$ em PVC, as amostragens foram realizadas seguindo ao grid anteriormente determinado (Figura 02), o quadro foi lançado aleatoriamente 8 vezes em cada ponto amostral. Posteriormente foi feita identificação das amostras, secagem natural das amostras, debulha e pesagem das amostras (OLIVEIRA, 2013). Em seguida os dados foram convertidos de $\mathrm{Kg} / \mathrm{m}^{2}$ para Ton./ha (MOLIN e MASCARIN, 2007). Além disso, a determinação do Peso de mil aquênios (PMA) foi feita de acordo com método relatado por BRASIL (2009b).

Os dados foram submetidos à análise exploratória, para obtenção das medidas de posição (média e mediana), medida de dispersão (desvio-padrão) e medidas de forma da distribuição (coeficiente de variação), avaliado de acordo com (Gomes, 2000), sendo também realizadas análises de correlação de Pearson e teste de normalidade, verificada através dos testes de Anderson Darling e Kolmogorov Smirnov (5\% de significância).

Para elaboração dos mapas temáticos da área experimental foi utilizado o software ArcGIS, onde os dados foram interpolados utilizando o interpolador inverso do quadrado da distância (IQD), interpolador determinístico univariado de médias ponderadas, ou seja, quanto mais distante um ponto observado estiver do estimado, menor será sua influência sobre o valor de inferência. Este método é considerado de acurácia satisfatória quando comparado a krigagem, podendo, em alguns casos, apresentar resultados semelhantes (SOUZA et al., 2010). Além disso, a similaridade dos mapas temáticos construídos a partir dos dados coletados foi avaliada por meio de análise visual dos mapas.

\section{RESULTADOS E DISCUSSÃO}

De acordo com a Tabela 1 nota-se que a produtividade média mostrou-se satisfatória, visto que segundo a Embrapa (2018) a média de produção brasileira é de $1300 \mathrm{~kg} / \mathrm{ha}$, o peso de 1000 sementes segundo o mesmo autor é de $_{[42} 50$ a $65 \mathrm{~g}$ e mostrou-se um pouco abaixo do esperado para espécie. Já em relação a análise de solo. pode-se observar que de acordo com 
interpretação proposta por Sousa e Lobato (2004) para os níveis de fertilidade do solo no bioma do cerrado, os valores médios dos atributos químicos, apresentaram-se muito baixo para $\mathrm{P}$, baixos nos atributos $\mathrm{K}, \mathrm{Ca}, \mathrm{M} \%, \mathrm{Zn}, \mathrm{Mn}, \mathrm{Fe}$ e $\mathrm{Cu}$, adequadas para $\mathrm{pH}, \mathrm{Mg}$ e $\mathrm{M} . \mathrm{O}$., médio para CTC, B e alto para V\%.

Além disso, de acordo com a média da resistência do solo à penetração não apresentou valores altos que possam afetar o estabelecido para o desenvolvimento radicular das plantas, segundo Canarache (1990) valores acima de 2,5 MPa começam a restringir o pleno crescimento das raízes da maioria das plantas.

Tabela 01: Análise estatística descritiva dos atributos avaliados.

\begin{tabular}{cccccccccc}
\hline Atributos & Máx. & Min. & Méd. & Medi. & D.P. & C.V. & Ass. & Cur. & Norm. \\
\hline $\mathrm{pH}$ & 5,85 & 4,45 & 5,23 & 5,26 & 0,53 & 10,06 & $-0,19$ & $-1,42$ & Sim \\
\hline $\mathrm{P}\left(\mathrm{mg} / \mathrm{dm}^{3}\right)$ & 3,21 & 0,09 & 0,98 & 0,43 & 1,06 & 108,37 & 1,26 & 0,34 & Sim \\
\hline $\mathrm{K}\left(\mathrm{mg} / \mathrm{dm}^{3}\right)$ & 11,70 & 7,80 & 8,78 & 7,80 & 1,76 & 20,10 & 1,33 & $-0,33$ & Não \\
\hline $\mathrm{Ca}\left(\mathrm{cmol}_{\mathrm{c}} / \mathrm{dm}^{3}\right)$ & 3,70 & 0,90 & 1,36 & 1,15 & 0,75 & 55,31 & 3,24 & 10,88 & Não \\
\hline $\mathrm{Mg}\left(\mathrm{cmol}_{\mathrm{c}} / \mathrm{dm}^{3}\right)$ & 1,20 & 0,70 & 0,91 & 0,85 & 0,17 & 18,46 & 0,96 & $-0,36$ & Sim \\
\hline $\mathrm{Al}\left(\mathrm{cmol}_{\mathrm{c}} / \mathrm{dm}^{3}\right)$ & 0,69 & 0 & 0,16 & 0 & 0,27 & 164,67 & 1,40 & 0,47 & Não \\
\hline $\mathrm{H}+\mathrm{Al}\left(\mathrm{cmol} / \mathrm{dm}^{3}\right)$ & 1,80 & 1,00 & 1,36 & 1,40 & 0,24 & 17,88 & 0,22 & $-0,78$ & Sim \\
\hline $\mathrm{SB}\left(\mathrm{cmol} / \mathrm{dm}^{3}\right)$ & 4,92 & 1,72 & 2,29 & 1,98 & 0,87 & 37,89 & 2,96 & 9,38 & Não \\
\hline $\mathrm{T}\left(\mathrm{cmol} / \mathrm{dm}^{3}\right)$ & 6,12 & 3,02 & 3,65 & 3,52 & 0,82 & 22,53 & 2,85 & 9,00 & Não \\
\hline $\mathrm{V} \%$ & 80,40 & 51,70 & 61,60 & 59,80 & 8,39 & 13,63 & 1,13 & 0,94 & Sim \\
\hline $\mathrm{M} \%$ & 27,70 & 0 & 6,73 & 0 & 10,78 & 160,29 & 1,27 & 0,01 & Não \\
\hline $\mathrm{MO}\left(\mathrm{g} / \mathrm{dm}^{3}\right)$ & 20,22 & 9,84 & 14,17 & 13,53 & 3,37 & 23,80 & 0,51 & $-0,95$ & Sim \\
\hline $\mathrm{Fe}\left(\mathrm{mg} / \mathrm{dm}^{3}\right)$ & 6,14 & 2,69 & 4,45 & 4,46 & 0,94 & 21,14 & $-0,05$ & 0,13 & Sim \\
\hline $\mathrm{Cu}\left(\mathrm{mg} / \mathrm{dm}^{3}\right)$ & 0,03 & 0,01 & 0,02 & 0,02 & 0,01 & 31,49 & $-0,06$ & 0,65 & Não \\
\hline $\mathrm{Zn}\left(\mathrm{mg} / \mathrm{dm}^{3}\right)$ & 0,09 & 0,01 & 0,05 & 0,06 & 0,03 & 66,05 & $-0,06$ & $-2,03$ & Sim \\
\hline $\mathrm{B}\left(\mathrm{mg} / \mathrm{dm}^{3}\right)$ & 0,38 & 0,29 & 0,35 & 0,36 & 0,02 & 7,10 & $-1,15$ & 2,14 & Sim \\
\hline $\mathrm{Mn}\left(\mathrm{mg} / \mathrm{dm}^{3}\right)$ & 1,83 & 0,54 & 1,01 & 0,94 & 0,44 & 43,43 & 0,55 & $-0,88$ & Sim \\
\hline $\mathrm{Prod} .\left(\mathrm{Ton} / \mathrm{ha}^{3}\right)$ & 2,62 & 0,56 & 1,71 & 1,89 & 0,80 & 47,03 & $-0,29$ & $-1,73$ & Sim \\
\hline $\mathrm{PMA}(\mathrm{g})$ & 49,60 & 30,70 & 41,38 & 42,90 & 5,95 & 14,37 & $-0,67$ & 0,08 & Sim \\
\hline $\mathrm{R}(\mathrm{Mpa})$ & 0,70 & 0,31 & 0,46 & 0,43 & 0,13 & 28,93 & 0,73 & $-0,80$ & Sim \\
\hline $\mathrm{U} \%$ & 6,90 & 5,60 & 6,23 & 6,20 & 0,41 & 6,61 & $-0,05$ & $-0,75$ & Sim \\
\hline & & & & & & & & \\
\hline
\end{tabular}


*pH - potencial Hidrogeniônico; P - Fósforo; K - Potássio; Ca - Cálcio; Mg - Magnésio; Al - Alumínio; H + Al Hidrogênio + Alumínio; SB - Soma de bases; T - Capacidade de Troca de Cátions à pH 7; MO - Matéria Orgânica; M\% - Saturação por Alumínio; V \% - Saturação de Bases; B - Boro; Mn - Manganês; Fe - Ferro; Cu Cobre; Zn - Zinco; Prod. - Produtividade; PMA - Peso de mil aquênios; U\% - Umidade do solo; R Resistência do solo a penetração; Mín - Mínimo; Máx. - Máximo; Méd. - Média; Medi. - Mediana; D.P. - Desvio padrão; C.V. - Coeficiente de variação; Curt. - Curtose; Ass. - Assimetria; Norm. - Normalidade, verificada através dos testes de Anderson Darling e Kolmogorov Smirnov (5\% de significância).

Fonte: Própria (2020).

Segundo Dalchiavon et al. (2012) a variabilidade de um atributo pode ser classificada conforme a magnitude de seu coeficiente de variação $(\mathrm{CV})$ como baixo $(\mathrm{CV}<10 \%)$, médio $(10 \%<\mathrm{CV}<20 \%)$, alto $(20 \%<\mathrm{CV}<30 \%)$ e muito alto $(\mathrm{CV}>30 \%)$. Com base nos resultados apresentados na Tabela 01, pode-se observar que a umidade do solo e o $\mathrm{B}$ apresentaram $\mathrm{CV}$ baixo, e CV médio para os atributos $\mathrm{pH}, \mathrm{H}+\mathrm{Al}, \mathrm{V} \%, \mathrm{Mg}$ e PMA, já para os demais atributos avaliados o coeficiente de variação foram classificados alto a muito alto, mostrando que o conjunto de dados apresenta heterocedasticidade.

De acordo com trabalho realizado por Zonta et al. (2014) e por Bottega et al. (2015), onde obtiveram comportamento semelhante sobre a fertilidade do solo. Do ponto de vista de Carvalho, Takeda e Freddi (2003), isto ocorre porque a variabilidade espacial dos atributos do solo pode ser influenciada pelos seus fatores de formação, que são o material de origem, relevo, clima, organismos e tempo, e pelas práticas de manejo do solo (adubação, calagem, dentre outros).

Segundo Machado et al. (2004), a variabilidade da fertilidade do solo pode ser consequência do manejo de adubação e da forma de cultivo implantado na área. Schlindwein e Anghinoni (2000) em seu trabalho sobre variabilidade espacial de atributos de fertilidade e amostragem de solo no sistema plantio direto, também encontraram maior variabilidade horizontal para fósforo e potássio. Zonta et. al. (2014), explicam que a alta variabilidade de teores $\mathrm{P}$ nos solos pode ser justificada, pelo fato da adubação ser realizada na linha de plantio o que resulta na desuniformidade na área e em razão da baixa mobilidade deste nutriente no solo.

Já sobre os atributos físicos do solo, resultados semelhantes foram relatados por Campos et al. (2012) e por Souza et al. (2006). Segundo relata Silva et al. (2004) na superfície e até a profundidade de $12 \mathrm{~cm}$, o solo sofre os maiores efeitos do tráfego de máquinas, da atividade biológica e de ciclos de umedecimento e secagem que provocam grande variabilidade e dispersão dos valores de Resistị̣̂̂pia do solo à penetração.

Além disso, a produtividade também apresentou coeficiente de variação alto, 
indicando comportamento heterogêneo. De acordo com Thylén et al. (1997), explicam que tal variabilidade pode ser resultado de diversos fatores que podem interferir na produção da cultura.

De acordo com a Tabela 02, pode-se observar o coeficiente de correlação de Pearson (r) entre os atributos de fertilidade do solo das 2 análises. De acordo com Figueiredo Filho e Silva Júnior (2009) uma correlação perfeita (-1 ou 1) indica que a contagem de uma variável pode ser determinada exatamente ao se saber a contagem da outra, no outro oposto, uma correlação de valor zero indica que não há relação linear entre as variáveis. Para interpretação das análises do coeficiente de correlação utilizou-se a proposta estabelecida por Dancey e Reidy (2006).

Conforme demonstra na Tabela 02, pode-se observar que dentre os atributos avaliados houve correlação entre a produtividade com os atributos de $\mathrm{PMA}, \mathrm{U} \%, \mathrm{pH}, \mathrm{H}+\mathrm{Al}, \% \mathrm{M}, \mathrm{B}, \mathrm{Fe}$, Mn e Zn, e também entre o Peso de mil aquênios e as variáveis $\mathrm{pH}, \mathrm{P}, \mathrm{Mg}, \mathrm{H}+\mathrm{Al}, \% \mathrm{~V}, \mathrm{~T}, \mathrm{MO}$, B, Fe, Mn e Zn. Resultado similar foi relatado por Pivetta et al. (2012) e por Strojaki et al. (2013), no qual os autores relataram correlação significativa entre a produtividade e o peso de mil aquênios.

Além disso na Tabela 02, nota-se também que houve correlação linear entre $\mathrm{P}$ e $\mathrm{K}$, correlação entre o pH com os atributos de U\%, Mg, Ca, V\%, H+Al, T, MO, M\%, B, Fe, Mn e Zn. Resultados semelhantes foram relatados por Junior et al. (2006), Montezano, Corazza e Muraoka (2006) e por Salviano, Vieira e Sparovek (1998). Segundo Gitti, Roscoe e Rizzato (2019), o baixo teor de $\mathrm{pH}$ do solo afeta a disponibilidade dos nutrientes essenciais para o desenvolvimento das plantas, e favorece a presença de elementos que inibem o desenvolvimento de raízes das plantas, tais como o Al.

Tabela 02: Coeficiente de correlação de Pearson entre os atributos avaliados. 


\begin{tabular}{|c|c|c|c|c|c|c|c|c|c|c|c|c|c|}
\hline PMA & 0,72 & & & & & & & & & & & & \\
\hline $\mathrm{R}$ & 0,16 & 0,20 & & & & & & & & & & & \\
\hline $\mathrm{U} \%$ & $\underline{0,46}$ & 0,12 & 0,22 & & & & & & & & & & \\
\hline $\mathrm{pH}$ & $\underline{0,53}$ & $\underline{0,46}$ & 0,27 & $\underline{0,35}$ & & & & & & & & & \\
\hline $\mathrm{P}$ & 0,18 & $\underline{-0,32}$ & $-0,24$ & $\underline{0,46}$ & 0,13 & & & & & & & & \\
\hline $\mathrm{K}$ & 0,21 & $-0,15$ & $\underline{-0,36}$ & $\underline{0,40}$ & 0,08 & 0,92 & & & & & & & \\
\hline $\mathrm{Ca}$ & $-0,10$ & 0,06 & $\underline{0,45}$ & $-0,12$ & $\underline{0,49}$ & 0,05 & $-0,10$ & & & & & & \\
\hline $\mathrm{Mg}$ & 0,27 & $\underline{0,42}$ & $\underline{0,34}$ & $-0,03$ & 0,69 & $-0,10$ & $-0,15$ & 0,64 & & & & & \\
\hline $\mathrm{H}+\mathrm{Al}$ & $\underline{-0,35}$ & $\underline{-0,57}$ & $-0,19$ & 0,09 & $-0,62$ & $\underline{0,54}$ & 0,60 & $-0,23$ & $-0,64$ & & & & \\
\hline $\mathrm{T}$ & $-0,14$ & $-0,03$ & $\underline{0,42}$ & $-0,09$ & $\underline{0,41}$ & 0,19 & 0,06 & $\mathbf{0 , 9 7}$ & 0,60 & $-0,05$ & & & \\
\hline V\% & 0,21 & $\underline{0,41}$ & $\underline{0,36}$ & $-0,09$ & 0,77 & $-0,22$ & $\underline{-0,32}$ & 0,77 & 0,91 & $-0,76$ & 0,66 & & \\
\hline $\mathrm{M} \%$ & $\underline{-0,56}$ & $\underline{-0,58}$ & 0,07 & $\underline{-0,33}$ & $-0,86$ & $-0,13$ & $-0,19$ & $-0,29$ & $\underline{-0,44}$ & $\underline{0,53}$ & $-0,20$ & $\underline{-0,57}$ & \\
\hline MO & $-0,07$ & $-0,22$ & $\underline{-0,51}$ & $\underline{0,43}$ & $\underline{-0,36}$ & $\underline{0,35}$ & $\underline{0,42}$ & $\underline{-0,43}$ & $\underline{-0,58}$ & $\underline{0,53}$ & $\underline{-0,36}$ & $-0,63$ & 0,05 \\
\hline \multicolumn{14}{|c|}{ Micronutrientes } \\
\hline & Prod. & PMA & $\mathrm{R}$ & \multicolumn{2}{|c|}{$\mathrm{U} \%$} & \multicolumn{2}{|c|}{ B } & \multicolumn{2}{|c|}{$\mathrm{Cu}$} & \multicolumn{2}{|c|}{$\mathrm{Fe}$} & \multicolumn{2}{|c|}{$\mathrm{Mn}$} \\
\hline B & $\underline{-0,59}$ & $\underline{-0,43}$ & $\underline{-0,47}$ & \multicolumn{2}{|c|}{$\underline{-0,49}$} & & & & & & & & \\
\hline $\mathrm{Cu}$ & $-0,25$ & $-0,16$ & 0,69 & \multicolumn{2}{|c|}{$-0,02$} & \multicolumn{2}{|c|}{$-0,19$} & & & & & & \\
\hline $\mathrm{Fe}$ & $\underline{-0,59}$ & $\underline{-0,41}$ & $-0,22$ & \multicolumn{2}{|c|}{$\underline{-0,35}$} & \multicolumn{2}{|c|}{$\underline{0,46}$} & \multicolumn{2}{|c|}{0,09} & & & & \\
\hline $\mathrm{Mn}$ & $\underline{-0,36}$ & $\underline{-0,51}$ & 0,08 & \multicolumn{2}{|c|}{$\underline{-0,34}$} & \multicolumn{2}{|c|}{$\underline{0,42}$} & \multicolumn{2}{|c|}{$\underline{0,37}$} & \multicolumn{2}{|c|}{0,10} & & \\
\hline $\mathrm{Zn}$ & $\underline{0,59}$ & $\underline{0,56}$ & $-0,12$ & \multicolumn{2}{|c|}{$\underline{0,47}$} & \multicolumn{2}{|c|}{$\underline{-0,44}$} & \multicolumn{2}{|c|}{$-0,16$} & \multicolumn{2}{|c|}{$\underline{-0,39}$} & \multicolumn{2}{|c|}{$\underline{-0,53}$} \\
\hline
\end{tabular}

$* \geq 0,60$ ou $r \geq-0,60$ (Correlação Forte - Em negrito); $0,30 \leq \mathrm{r}<0,60$ ou $-0,60<\mathrm{r} \leq-0,30$ (Correlação Moderada

- Em itálico e sublinhado); -0,30 $<\mathrm{r}<0,30$ (Correlação Fraca - Fonte normal) (Dancey e Reidy, 2006); pH potencial Hidrogeniônico; P - Fósforo; K - Potássio; Ca - Cálcio; Mg - Magnésio; Al - Alumínio; H + Al -

Hidrogênio + Alumínio; T - Capacidade de Troca de Cátions à pH 7; MO - Matéria Orgânica; M\% - Saturação por Alumínio; V \% - Saturação de Bases; B - Boro; Mn - Manganês; Fe - Ferro; Cu - Cobre; Zn - Zinco; Prod. Produtividade; PMA - Peso de mil aquênios; U\% - Umidade do solo; R - Resistência do solo a penetração.

Fonte: Própria (2020).

Nas Figuras 5,6 e 7 são apresentados os mapas da produtividade, peso de mil aquênios, resistência do solo à penetração, umidade e fertilidade do solo. As escalas dos mapas de fertilidade dos solos foram elaboradas segundo a interpretação proposta por Sousa e Lobato (2004), já a escala dos demais mapas foram padronizadas na divisão por quartis no ArcGIS, tendo por objetivo observar a variação dos mapas. 
Figura 05. Mapas de produtividade e PMA e dos atributos fisicos do solo da área experimental
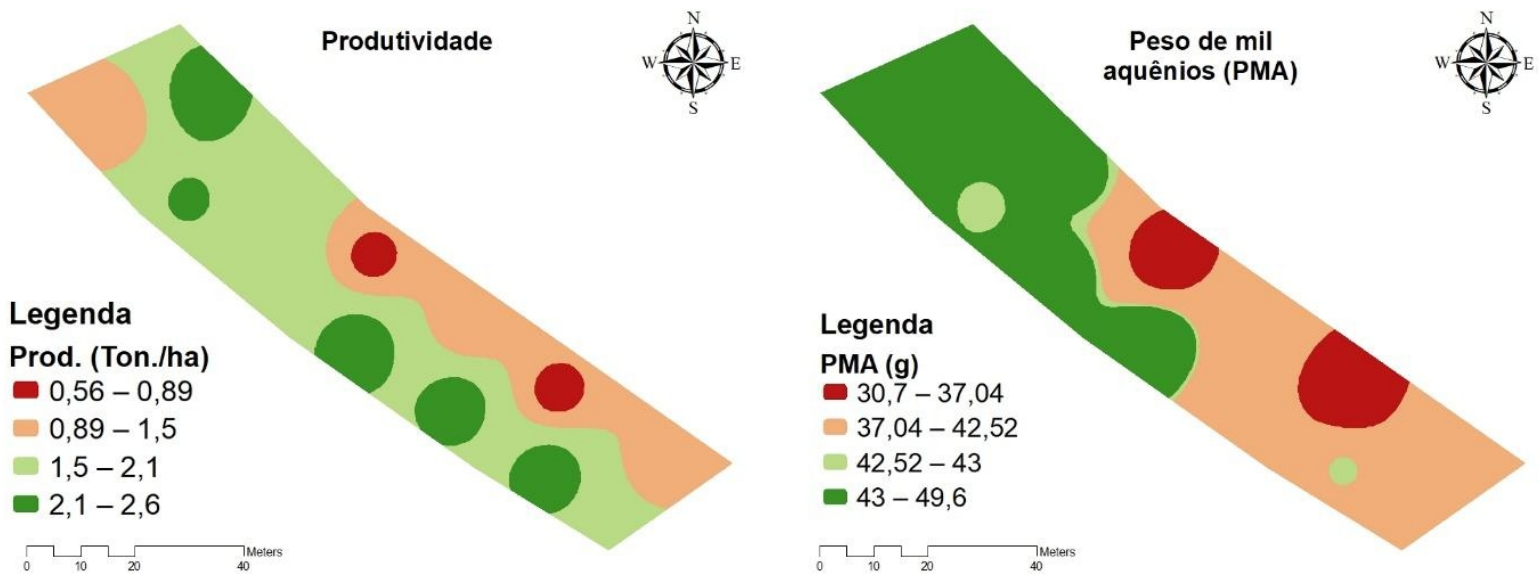

Fonte: Própria (2020).

Figura 06. Mapas de resistência do solo a penetração (RP) e umidade do solo
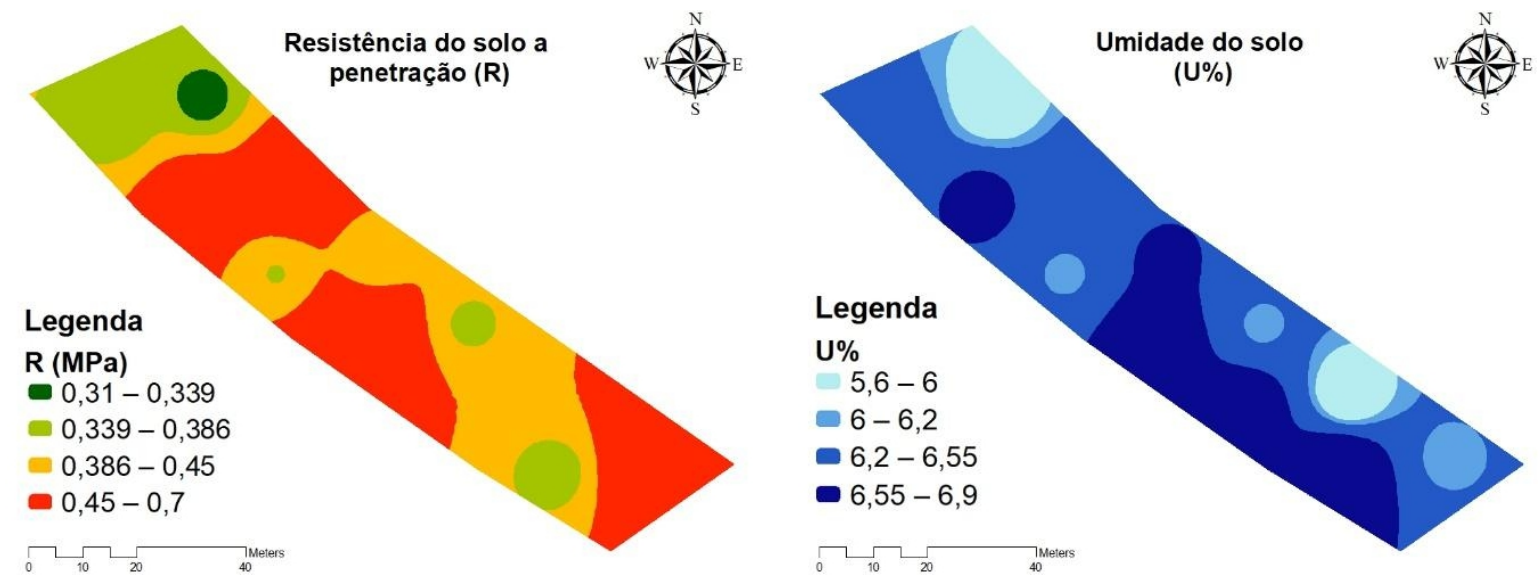

Fonte: Própria (2020).

Figura 07. Mapas de fertilidade do solo da área experimental. 


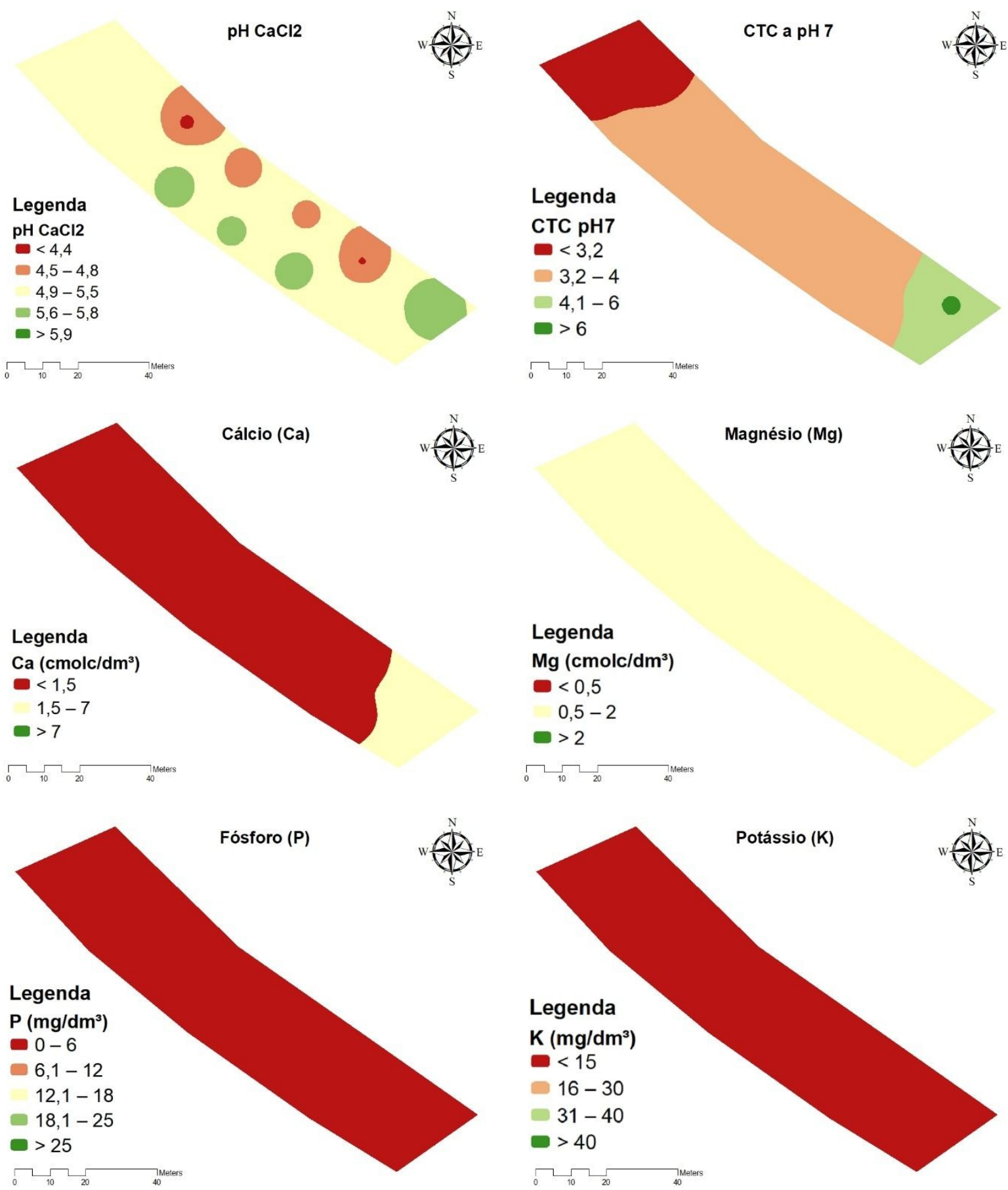

[48] 

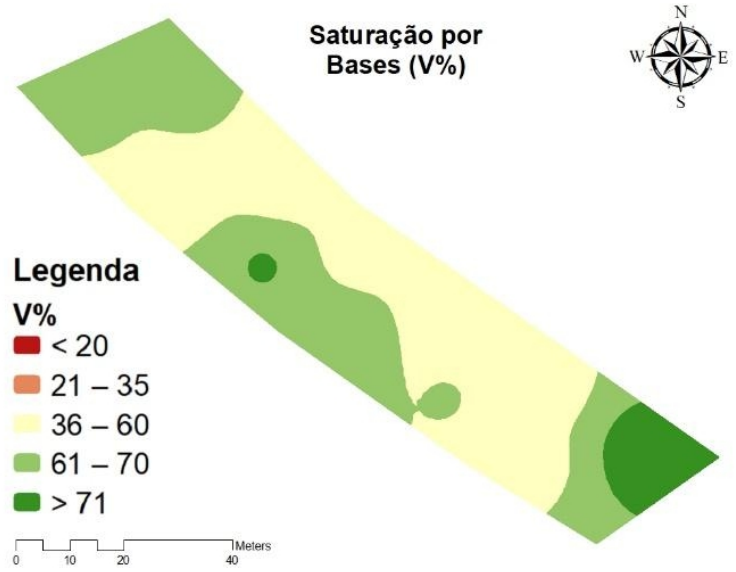

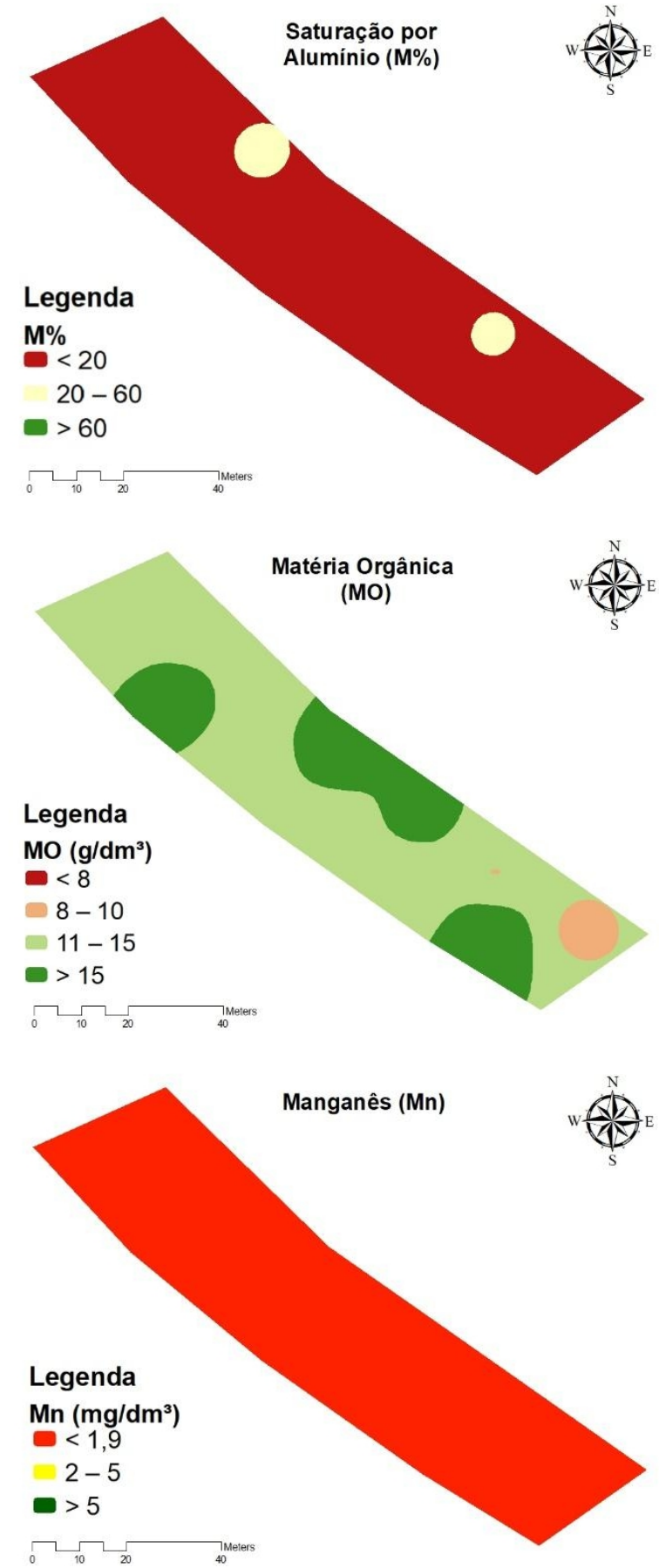

(MO)

[49]

\section{Legenda}

\section{B $\left(\mathrm{mg} / \mathrm{dm}^{3}\right)$}

- $<0,2$

$0,3-0,5$

- $>0,5$

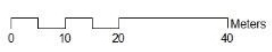

Boro (B)
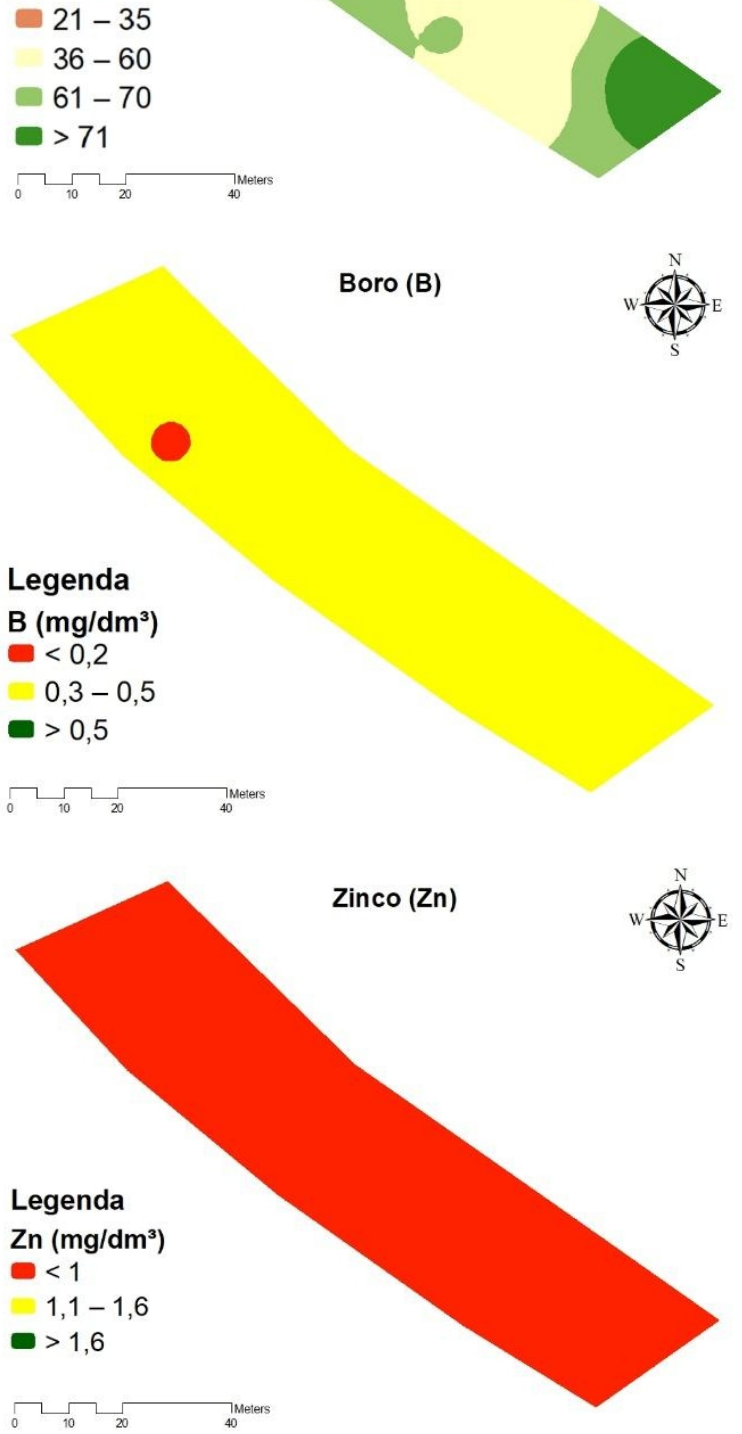

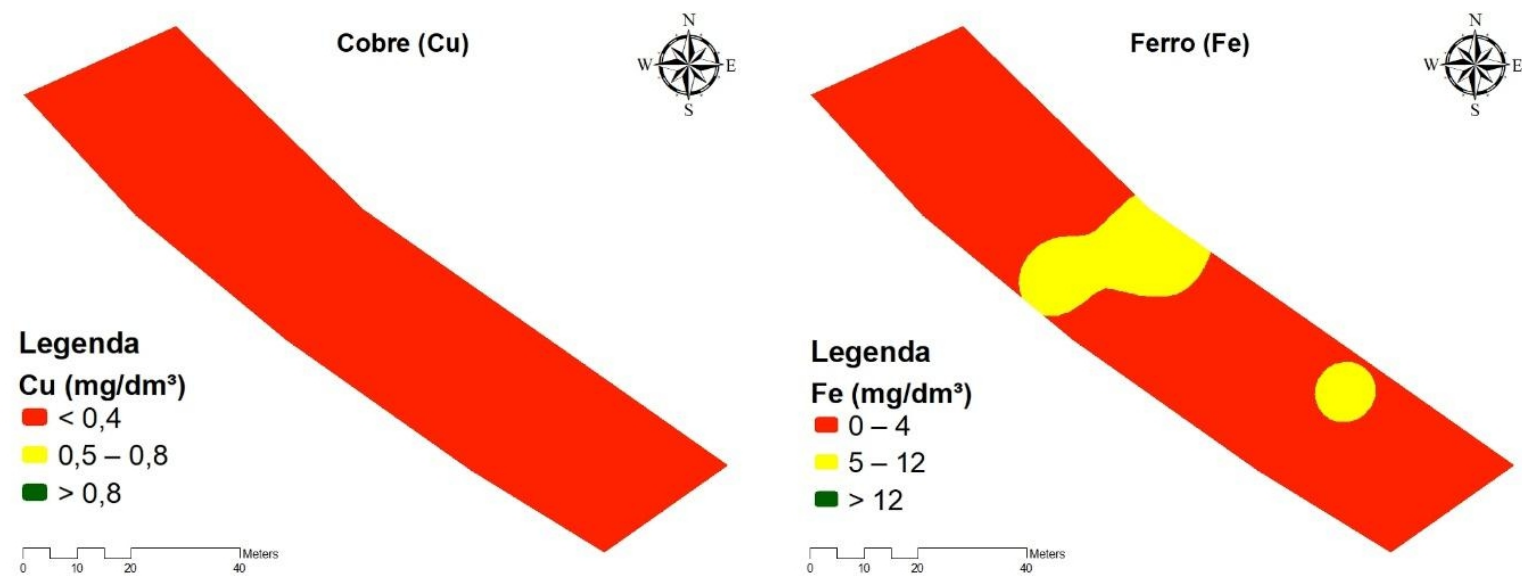

Fonte: Própria (2020).

Com base nos mapas apresentados nas Figura 05, 06 e 07, nota-se que não houve variabilidade espacial dos teores de $\mathrm{Mg}, \mathrm{P}, \mathrm{K}, \mathrm{Mn}, \mathrm{Zn}$ e $\mathrm{Cu}$ na área experimental, já nos mapas de CTC, B, M\%, Ca e Fe demonstrou baixa variabilidade espacial, e alta variabilidade espacial nos demais mapas. De maneira geral pode-se verificar que na região sudeste houve produtividade baixa, PMA baixo, altos valores de RP, baixa umidade do solo, altos teores de $\mathrm{V} \%$, teores adequados de $\mathrm{pH}, \mathrm{CTC}, \mathrm{Ca}, \mathrm{Mg}$ e $\mathrm{MO}$, concentração média de Boro, baixos níveis de $\mathrm{K}, \mathrm{M} \%, \mathrm{Mn}, \mathrm{Zn}, \mathrm{Cu}$ e $\mathrm{Fe}$, e teor muito baixo de Fósforo. Além disso na região central apresentou produtividade média, PMA baixo, valores médio de RP, alta umidade do solo, altos níveis de Matéria Orgânica, concentração adequada de $\mathrm{pH}, \mathrm{Mg}$ e V\%, nível médio de CTC e Boro, teores baixos de Ca, K, M\%, Mn, Zn, Cu e Fe, e nível muito baixo de Fósforo. Já na região nordeste nota-se predominância de produtividade alta, PMA alto, baixos valores de RP, médio teor umidade do solo, nível alto de $\mathrm{V} \%$, teores adequados de $\mathrm{pH}, \mathrm{Mg}$ e $\mathrm{MO}$, concentração média de Boro, baixos teores de CTC, Ca, K, M\%, Mn, Zn, Cu e Fe, e concentração muito baixa de Fósforo. Segundo Scapinelli, et al. (2016), valores maiores que $0,733 \mathrm{MPa}$ de resistência do solo à penetração promovem redução na produtividade do girassol, sendo considerados críticos para a cultura.

Além disso, de acordo com Cancian (2015), quando o teor de água aumenta, a resistência do solo a penetração tem seus valores diminuídos pela redução das forças de coesão que atuam entre as partículas de solo, o que ocasiona um aumento das forças de adesão mudando a consistência de friável para plástica, e desta forma, alcançando valores limitantes ao crescimento de raízes em condições de baixo teor de umidade no solo.

$$
\text { [50] }
$$

De acordo Sousa e Lobato (2004), o V\% adequado para cultura do girassol é entre 
$50 \%$ a $60 \%$, já segundo Castro et al. (1996), em valores de $\mathrm{pH}$ inferior a 5,2 a cultura do girassol apresenta sintomas de toxidez, devido a cultura apresentar sensibilidade à acidez do solo.

Além disso, com base na baixa variabilidade dos teores de potássio e fósforo apresentado nos mapas da Figura 07, pode-se afirmar que a adubação de correção desses nutrientes deve ser realizada em taxa única, tal resultado também foi relatado em Zonta et al. (2014).

\section{CONCLUSÕES}

Foi possível identificar variabilidade espacial na área experimental, através do mapeamento da produtividade e dos atributos físicos e químicos do solo. Além disso, observou-se correlação significativa entre a produtividade, o peso de mil aquênios e os atributos de $\mathrm{pH}, \mathrm{H}+\mathrm{Al}, \mathrm{B}, \mathrm{Fe}$ e $\mathrm{Mn}$.

\section{REFERÊNCIAS}

BELLETI, S.Z.Z. Variabilidade temporal e espacial de um latossolo vermelho em área comercial cultivada com Soja na região de Rondonópolis - MT. 2009. 73 f. Monografia (Agronomia) - Curso de graduação em Agronomia, Pontifícia Universidade Católica do Paraná-PR. 2009.

BERNARDI, A. C. C. et al. Agricultura de precisão: resultados de um novo olhar. $1^{\underline{a}}$ ed. Embrapa: Brasília-DF, 2014.

BOTTEGA, E.L.; QUEIROZ, D.M.; SANTOS, N.T.; PINTO, F.A.C.; SOUZA, C.M.A. Correlação entre condutividade elétrica aparente e atributos químicos e físicos de um Latossolo. Comunicata Scientiae, Bom Jesus, v.6, n.2, p.134-142, Abr./Jun. 2015.

BRASIL. Ministério da Agricultura, Pecuária e Abastecimento. Agricultura de precisão. Brasília-DF: Mapa/ACS, 2009a. 31 p.

BRASIL. Ministério da Agricultura, Pecuária e Abastecimento. Regras para análise de sementes. Brasília-DF: Mapa/ACS, 2009b. 399 p.

CANARACHE, A. Penetrometer - a generalized semi- empirical model estimating soil resistance to penetration. Soil Till Res., v. $16_{[\$ 51\}} 1-70,1990$. 
CANCIAN, L. C. Variabilidade espacial da resistência a penetração, granulometria e umidade do solo. 2015. 59 f. Dissertação (Mestrado em Agronomia) - Curso de Pósgraduação em Agronomia, Universidade Federal de Santa Maria-RS. 2015.

CAMPOS, M.C.C. et al. Variabilidade espacial da resistência do solo à penetração e umidade em áreas cultivadas com mandioca na região de Humaitá, AM. Revista Agro@mbiente On-line, v. 6, n. 1, p. 09-16, 2012.

CARVALHO, M.P.; TAKEDA E.Y. \& FREDDI, O.S. Variabilidade espacial de atributos de um solo sob videira em Vitória Brasil-SP. Revista Brasileira Ciências do Solo, v. 27 p. 695 703, 2003.

CASTRO, C. et al. A cultura do girassol. Circular técnica. 13, EMBRAPA-CNPSo. Londrina-PR; 38p. 1996.

CHERUBIN, M.R. et al. Amostragem de solo na agricultura de precisão. In: SANTI, L.A. Agricultura de Precisão no Rio Grande do Sul. 1ª Ed. CESPOL, Santa Maria-RS; 2016, p. 309.

CONAB. Acompanhamento da safra brasileira: Grãos, v. 6, safra 2018/2019, Quarto levantamento. Brasília-DF, 2019, p.126.

COMUNELLO, E.; SALTON, J. C.; TOMAZI, M.; AMARAL, S. S. W. G. Análise geoestatística da condutividade elétrica aparente do em sistemas de integração lavourapecuária em Mato Grosso do Sul. Agricultura de Precisão: Resultados de um novo olhar. EMBRAPA. Brasília-DF. 2014. cap. 56, p. 530-538.

DALCHIAVON, F.C. et al. Variabilidade espacial de atributos da fertilidade de um Latossolo Vermelho Distroférrico sob Sistema Plantio Direto. Revista Ciência Agronômica, v. 43, p. 453-461, 2012.

DANCEY, C.; REIDY, J. Estatística sem matemática para psicologia: usando SPSS para Windows. Porto Alegre-RS: Artmed, 2006.

FARIAS NETO, A. L.; ANDRADE, L. R. M.; OLIVEIRA, M. F. Metodologia para seleção de genótipos de girassol (Helianthus annuus L.) tolerantes ao alumínio. Planaltina: Embrapa-CPAC, 1999. 2 p. (Pesquisa em Andamento, 25).

FIGUEIREDO FILHO, D.B.; SILVA JÚNIOR, J.A. Desvendando os Mistérios do Coeficiente de Correlação de Pearson (r). Revista Política Hoje, v. 18, n. 1, 2009.

GAZZOLA, A. et al. A cultura do girassol. São Paulo: USP, 2012. 69 p.

GIMENEZ, L. MOLIN, J.P. Mapeamento da Produtividade. In: MACHADO, P.L.O.A.; BERNARDI, A.C.C., SILVA, C.A. Agricultura de precisão para o manejo da fertilidade do solo em sistema plantio direto. Rio de Janeiro : Embrapa Solos, 2004, p. 77-92.

GITTI, D.C.; ROSCOE, R.; RIZZATO, L.A. [\$2/hejo e fertilidade do solo para Soja. In:

LOURENÇÃO, A.L.F. et al. Tecnologia e produção: Safra 2018/2019. Maracaju: Midiograf. 
2019. 198 p.

GOMES, F.P. Curso de estatística experimental. 14ª ed. Piracicaba-SP, Degaspari. 2000. $477 \mathrm{p}$.

JUNIOR, A.R. et al. Variabilidade espacial e temporal de atributos químicos do solo e da produtividade da soja num sistema de agricultura de precisão. Engenharia na Agricultura, Viçosa-MG, Jul./Set, 2006 v.14, n.3, p.156-169.

LARK, R.M.; STAFFORD, J.V. Exploratory analysis of yield maps of combine crops. In: EUROPEAN CONFERENCE ON PRECISION AGRICULTURE, 1, 1997, Warwick.

Proceedings... Oxford: BIOS Scientific, 1997. p. 887-894.

LEITE, R. M. V. B. C. Doenças do girassol. In: AMORIM, L.; REZENDE, J. A. M.; BERGAMIN FILHO, A. (Ed.). Manual de fitopatologia: doenças das plantas cultivadas. 5․ ed. Ouro Fino: Agronômica Ceres, 2016. v. 2. p. 445- 461.

LIRA, M.A. et al. Desempenho de cultivares de girassol (Helianthus annuus. L.) no estado do Rio grande do norte. In: Congresso brasileiro de mamona, 4 \& Simpósio internacional de oleaginosas energéticas, 1., 2010, João Pessoa. Inclusão Social e Energia: Anais... Campina grande: Embrapa Algodão, 2010, p. 1700-1703.

MACHADO, P.L.O.A.; BERNARDI, A.C.C.; SILVA, C.A. Agricultura de precisão para o manejo da fertilidade do solo em sistema plantio direto. Rio de Janeiro: Embrapa Solos, 2004. 209p.

MOLIN, J.P.; MASCARIN, L.S. Colheita de citros e obtenção de dados para mapeamento da produtividade. Eng. Agrícola, Jaboticabal, v. 27, n.1, p. 259-266, jan./abr. 2007.

MOLIN, J.P.; AMARAL, L. R.; COLAÇO, A. F. Agricultura de precisão. São Paulo: Oficina de Textos, 2015. $238 \mathrm{p}$.

MONTEZANO, Z.F.; CORAZZA, E.J.; MURAOKA, T. Variabilidade espacial da fertilidade do solo em área cultivada e manejada homogeneamente. Revista Brasileira Ciências do Solo, v. 30, p. 839-847, 2006.

OLIVEIRA, C.M. Qualidade de sementes de girassol em função da posição no capítulo e da densidade de plantas. 2013. $60 \mathrm{f}$. Tese ( Doutorado em Agronomia) - Universidade federal de Uberlândia-MG. 2013.

OLIVEIRA, S. L. et al. Componentes Produtivos de genótipos de girassol cultivados em Januária, Norte de Minas. In: XXII Reunião Nacional de Pesquisa do girassol e X Simpósio Nacional sobre a cultura do Girassol; Lavras-MG. 2017.

PIVETTA, L. G. et al. Avaliação de híbridos de girassol e relação entre parâmetros produtivos e qualitativos. Revista Ciência Agronômica, Fortaleza-CE; v. 43, n. 3, p. 561-568 2012.

[53] 
RIBAS, M.L. Caracteres agronômicos de cinco genótipos de Girassol (Helianthus annuus L.) avaliados em campos dos Goytacazes - RJ. 2009. 58 f. Monografia (Mestrado em Produção vegetal) - Universidade Estadual do Norte Fluminense Darcy Ribeiro-RJ. 2009.

SALVIANO, A.A.C.; VIEIRA, S.R. \& SPAROVEK, G. Variabilidade espacial de atributos de

solo e de Crotalaria juncea L. em área severamente erodida. Revista Brasileira Ciências do. Solo, v. 22, p. 115-122, 1998.

SANTOS, H. G. et al. Sistema Brasileiro de Classificação de Solos. 5a ed. Brasília: 2018, p. 355.

SCHLINDWEIN, J. A.; ANGHINONI, I. Variabilidade espacial de atributos de fertilidade e amostragem de solo no sistema plantio direto. Revista Brasileira de Ciência de Solo, Viçosa-MG, v. 24, n.1, p. 85-91, 2000.

SILVA, V.R. et al. Variabilidade espacial da resistência do solo à penetração em plantio direto. Ciência Rural, Santa Maria, v. 34, n. 2, p. 399-406, 2004.

SOUSA, D.M.G.; LOBATO E. CERRADO correção do solo e adubação. 2ª ed. Brasília-DF: Embrapa Informação tecnológica, 2004, p. 416.

SOUZA, G.S. et al. Krigagem ordinária e inverso do quadrado da distância aplicados na especialização de atributos químicos de um argissolo. Scientia Agraria, Curitiba-PR, v. 11, n. 1, p. 73-8, 2010.

SOUZA, Z.M. et al. Dependência espacial da resistência do solo à penetração e do teor de água do solo sob cultivo contínuo de cana-de-açúcar. Ciência Rural, Santa Maria, v. 36, n.1, p.128-134, 2006.

STROJAKI, T.V. et al. Atributos químicos do solo e produtividade de girassol e milho em função da aplicação de composto de lixo urbano. Pesq. Agropec. Trop., Goiânia, v. 43, n. 3, p. 278-285, jul./set. 2013.

THYLÉN, L.; JURSCHIK, P.; MURPHY, D. L. P. Improving the quality of yield data, In: EUROPEAN CONFERENCE ON PRECISION AGRICULTURE, 1a ed., 1997, Warwick. Proceedings... Oxford: BIOS Scientific, 1997. p. 743-750.

ZONTA, J.H. et. al. Variabilidade espacial da fertilidade do solo em área cultivada com algodoeiro no Cerrado do Brasil. Revista Brasileira de Engenharia Agrícola e Ambiental, v.18, n. 6, p. 595-602, 2014. 